\title{
Infinitely adjustable attenuation cell for infrared radiation to fit the metering capacity to different object temperatures
}

\author{
Rademacher, Hans-Georg \\ TU Dortmund / Chair of Quality \\ Joseph-von-Fraunhofer-Str. 20 \\ D-44227 Dortmund \\ Germany
}

\section{Introduction}

In this work a method is described, which allows the extension of measurement prospects of the metering capacity of an infrared camera system. In the following examples a high-speed infrared camera with a MCT-sensor (3-5 $\mu \mathrm{m}$ spectral response) and a frame rate of 885 pictures per seconds is used. This type of camera is not equipped with temperature measurement like standard thermography cameras. Its major task is to detect small changes in the radiation intensity. To match the measurement-range of the camera to the amount of emitted radiation by the inspected object, the only parameter needed to change is the exposure time. The few possible discrete steps of changing this time constant, which most cameras contain, can extend the measurement range only to a maximal object temperature, which corresponds to a maximal intensity that can be measured. If the temperature increases the detector will overcharge. This also affects neighbouring pixels (blooming effect). Furthermore the used camera has a limitation when changing the exposure time. After each change, a sensitivity calibration and a homogenizing of the MCTSensor is necessary. This could be difficult if the camera is attached in a complicated assembling and makes a comparison of different measurements very difficult. Another disadvantage is the decreasing sensitivity when using short exposure times. The camera is in fact able to detect higher changes in temperature, but concurrently it is less sensitive to objects at lower temperatures. The measuring of two objects with a great difference in temperature, which appears for example at cutting processes, is difficult or not possible. As well, focusing the object under test is difficult at room temperature, as the contours cannot be seen.

\section{Solution statement}

As described above the following problems should be solved:

- Extension of a given metering capacity

- Prevention of overcharging the sensor

- Prevention of the blooming effect

- Prevention of changing the exposure time

- Measuring of two different temperature ranges at the same time

These problems can be solved by using attenuation cells and radiation stops. From the classical optical design neutral density filters are known. These filters work only in the visible wavelengths region till the near infrared. The used glass substrate doesn't work between $3 \mu \mathrm{m}$ and $5 \mu \mathrm{m}$. Here materials like germanium, calcium fluoride or sapphire are the first choice. In the spectral range from $8 \mu \mathrm{m}$ to $12 \mu \mathrm{m}$ some kind of plastics work fine, too. By varying the thickness of a proper substrate the amount of attenuation can be adjusted. In fact there is an amount of filters necessary for this assignment. To infinitely adjust the attenuation another physical method is used here. A polarizer polarizes light, which is normally random polarized. When this polarized light passes a second polarizer, the resulting intensity $\mathrm{I}_{\mathrm{P} 1}$ of the light $\mathrm{I}_{\mathrm{P} 2}$ depends on the angle $\Theta$ between the axes of the polarizers (Fig. 1). In special cases the light is already (partly) polarized - for example after reflected at a surface. Then only one polarizer suffices to vary the intensity mainly from the reflected object. Thereby nuisance reflections can be localized and reduced (Fig. 6). For this application the polarizer must of course be transparent for the infrared radiation. Instead of expensive wire grid polarizers a new kind of polarizer based on silver nanoparticles is used. This type of filter can easily be structured to polarize different regions in the picture in different ways. 


\section{Experimental Setup}

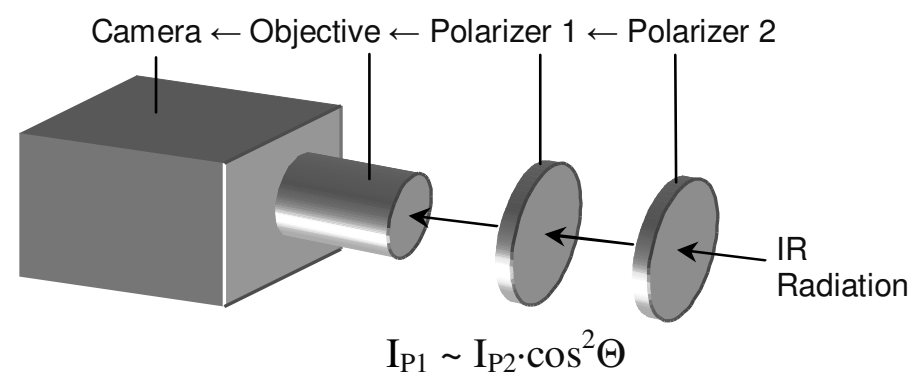

Fig. 1: Schematic view of the infinitely adjustable attenuator cell consisting of two polarizers.

To verify the function of the described attenuator cell the schematic shown in Fig. 1 was transferred to an experimental setup shown in Fig. 2. Polarizer 1 is placed between the MCT-Detector and the objective. Therefore only polarized light can reach the detector. So far this assembly is also used for the experiment to reduce nuisance radiation from reflection. The tap of the soldering iron will be observed through Polarizer 2, which is placed on an attachment. In Fig. 3 the taken infrared-images from this experiment are shown. In the left the soldering iron is seen without polarizer 2. Through the attachment the tap of the soldering iron becomes visible. The temperature is about $300^{\circ} \mathrm{C}$ and a small blooming effect is visible because of the detectors overcharging. In the middle the tap is clearly visible without blooming. This appears now only at the heating element between the handhold and the tap and could be shielded with a radiation stop. With this arrangement the aim of measuring two temperatures (nearly room temperature and about $300^{\circ} \mathrm{C}$ ) is reached. In the right part of the figure the soldering iron is heated up to $450^{\circ} \mathrm{C}$. The tap is still clearly visible and the blooming effect at the heater has increased.

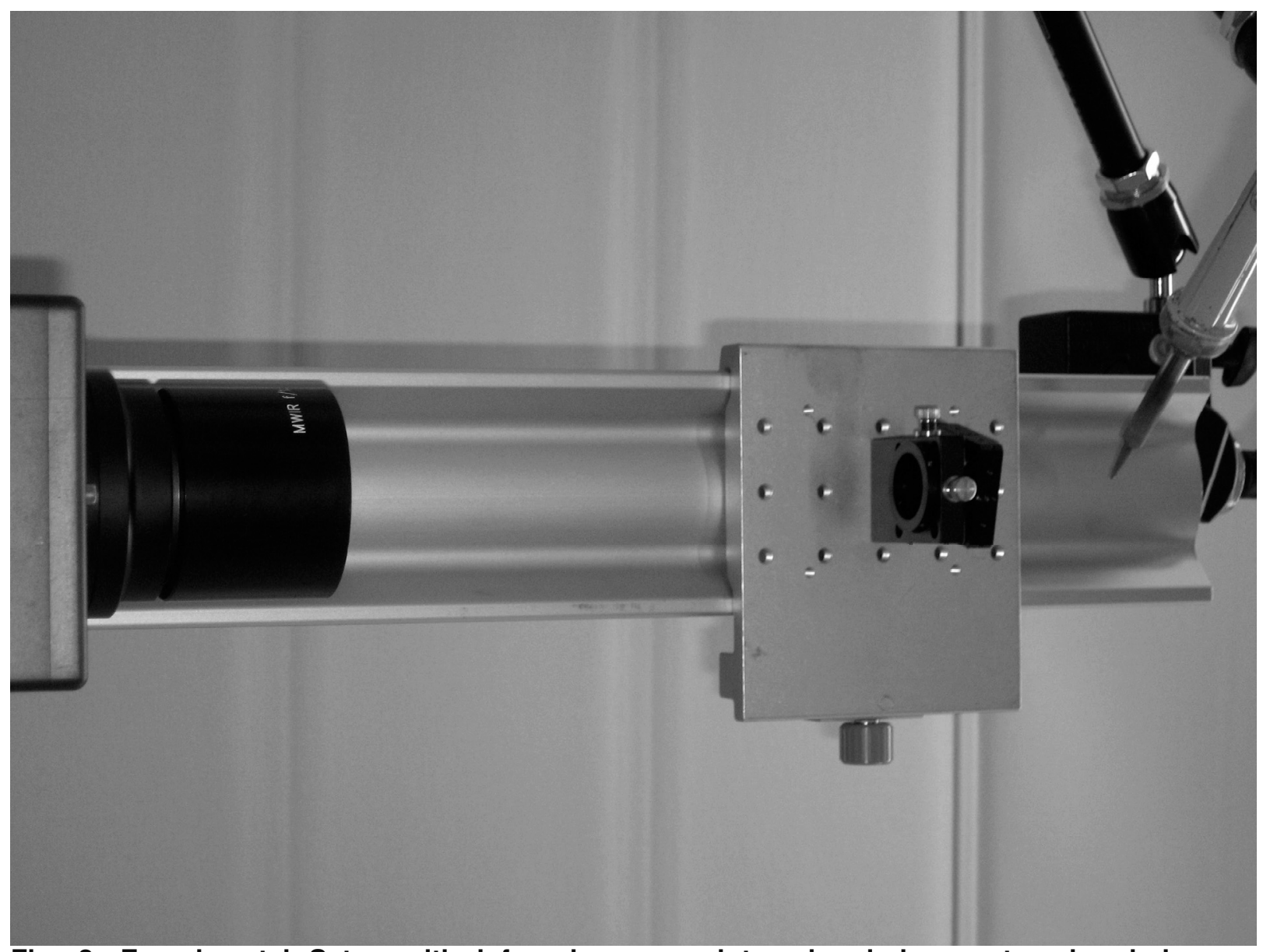

Fig. 2: Experimental Setup with infrared camera, internal polarizer, external polarizer and Soldering Iron to observe. 


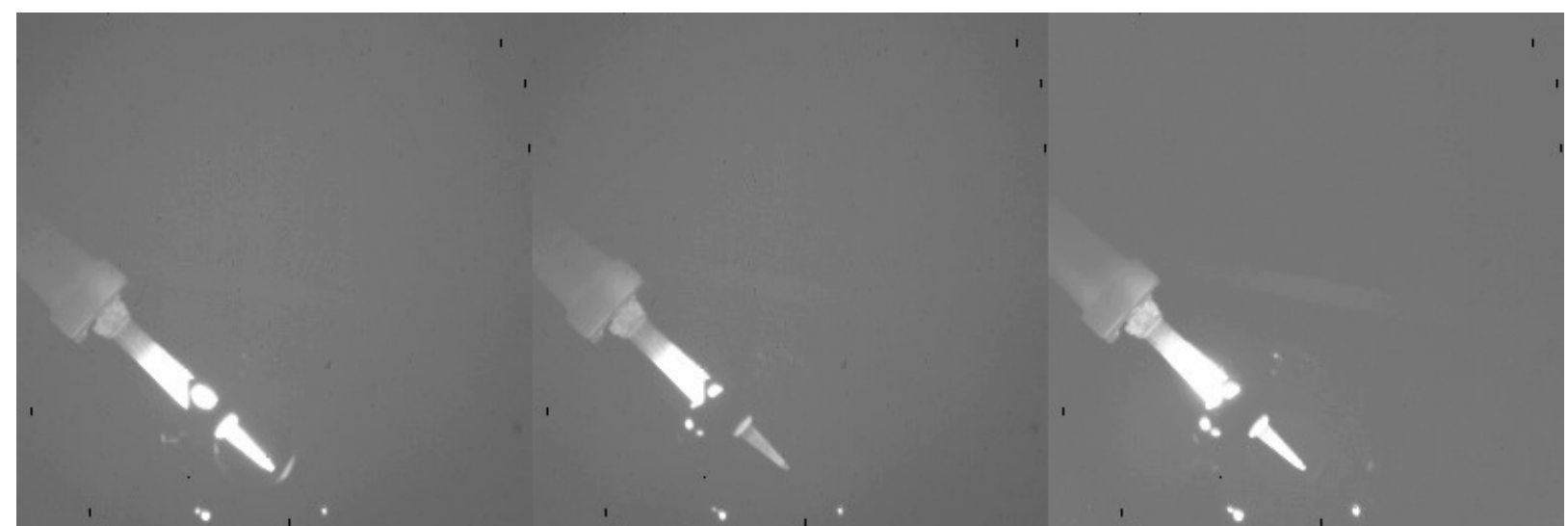

Fig. 3: Infrared Image of a Soldering Iron without attenuator @ $300^{\circ} \mathrm{C}$ (left), with attenuator @ $300^{\circ} \mathrm{C}$ (middle) and with attenuator @ $450^{\circ} \mathrm{C}$ (right)

The differences which are shown in Fig. 3 fall into place with the diagram shown in Fig. 4. The measured intensity of the soldering iron tap is plotted over the time. The grey line represents the intensity without attenuator. In this case the detector is overcharged and the temperature difference of $150^{\circ} \mathrm{C}$ causes a signal change of about 30 digits. In the case with the attenuator, the absolute intensity level is much lower but the process of heating up from $300^{\circ} \mathrm{C}$ to $450^{\circ} \mathrm{C}$ and back to $300^{\circ} \mathrm{C}$ is clearly visible. Also the transient response can be observed. Now the signal deviation is about 1000 digits.

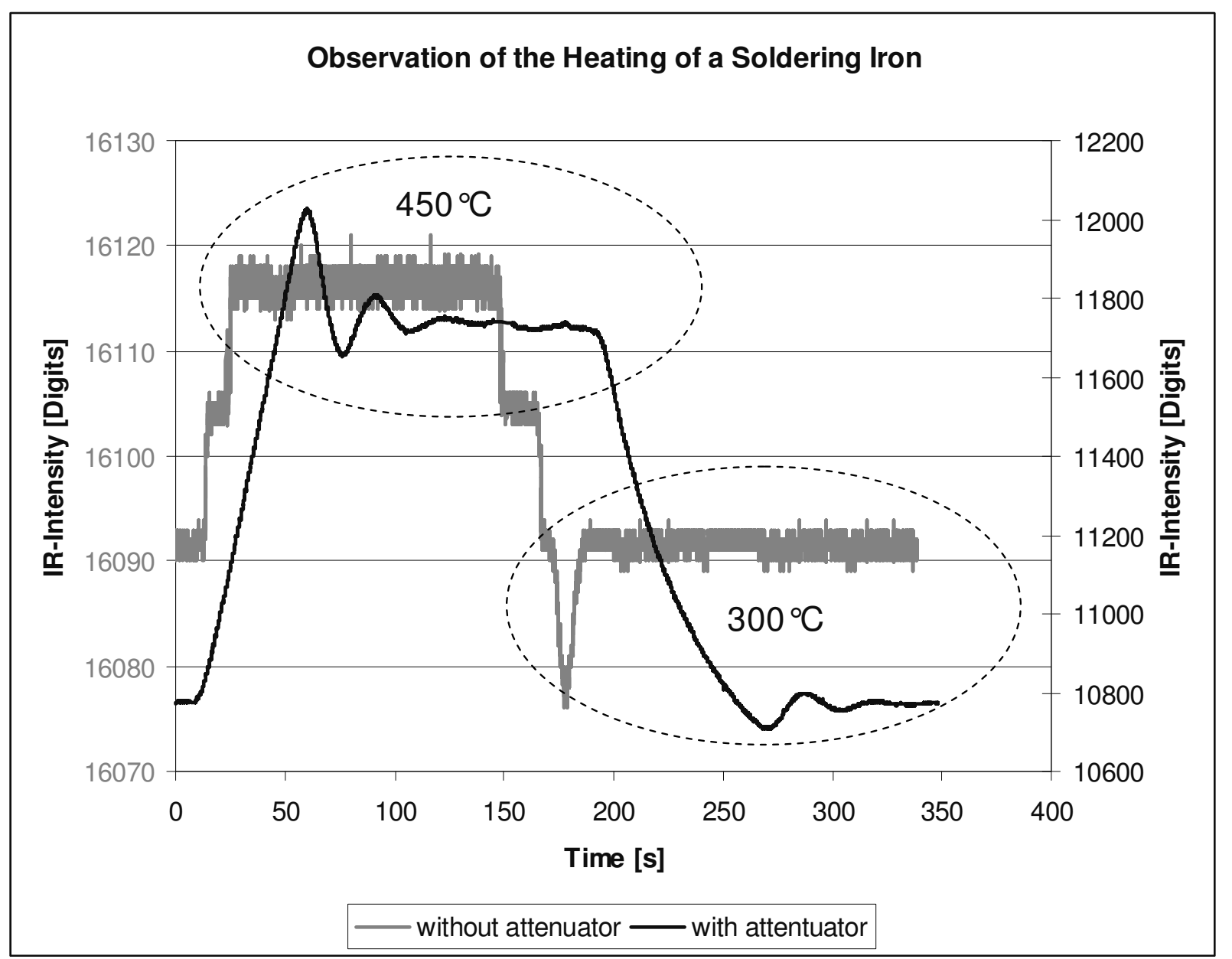

Fig. 4: Measured IR-Intensity of a Soldering Iron at $450^{\circ} \mathrm{C}$ and $300^{\circ} \mathrm{C}$ without attenuator (grey chart) and with attenuator (black chart) 
The second experimental setup is a variation of the first one. Here the polarizer 2 is missing (Fig. 5). The black body on the right is set to $150^{\circ} \mathrm{C}$. Its radiation is reflected by the polished surface of a silicon-wafer on the left. The whole scene is observed by the camera through the polarizer 1 between the detector and the objective. By rotating the polarizer 1 a minimum and a maximum intensity of the reflected radiation was found. These two stages are shown in Fig. 6. On the left the intensity of the big sphere (the direct radiation from the black body) is a little higher than on the right side. With the indirect radiation, reflected at the silicon-wafer, it's vice versa. On the left the intensity is much lower. This result is also shown by the measured values in Fig. 7. The intensity change of the reflected radiation is significant higher $(6,6 \%)$ compared to the change of the direct radiation $(0,8 \%)$. It's herewith certified that it is possible to reduce nuisance reflections and detect them. Similar to the use in photography the effect depends on the surface properties and the used material.

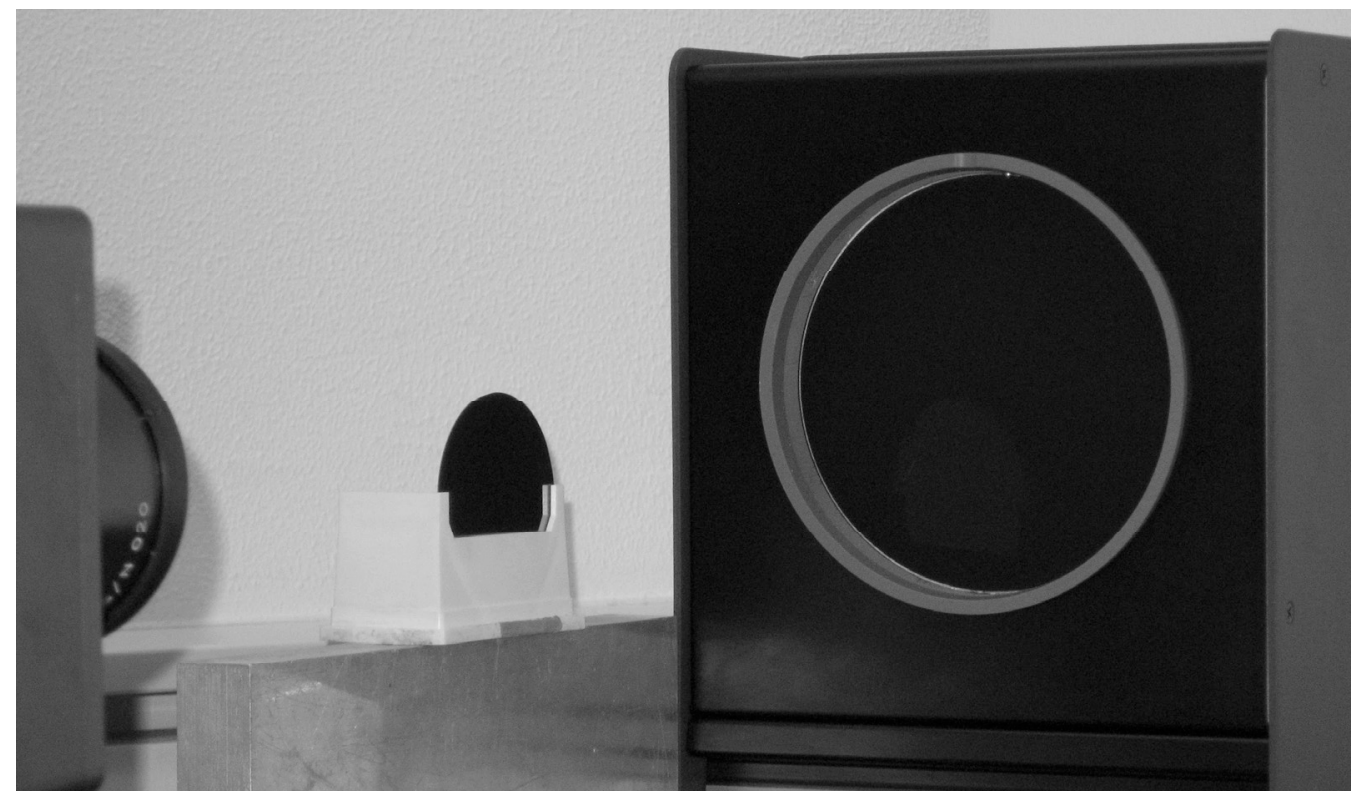

Fig. 5: Experimental Setup with infrared camera, internal polarizer, Black Body and siliconreflector.

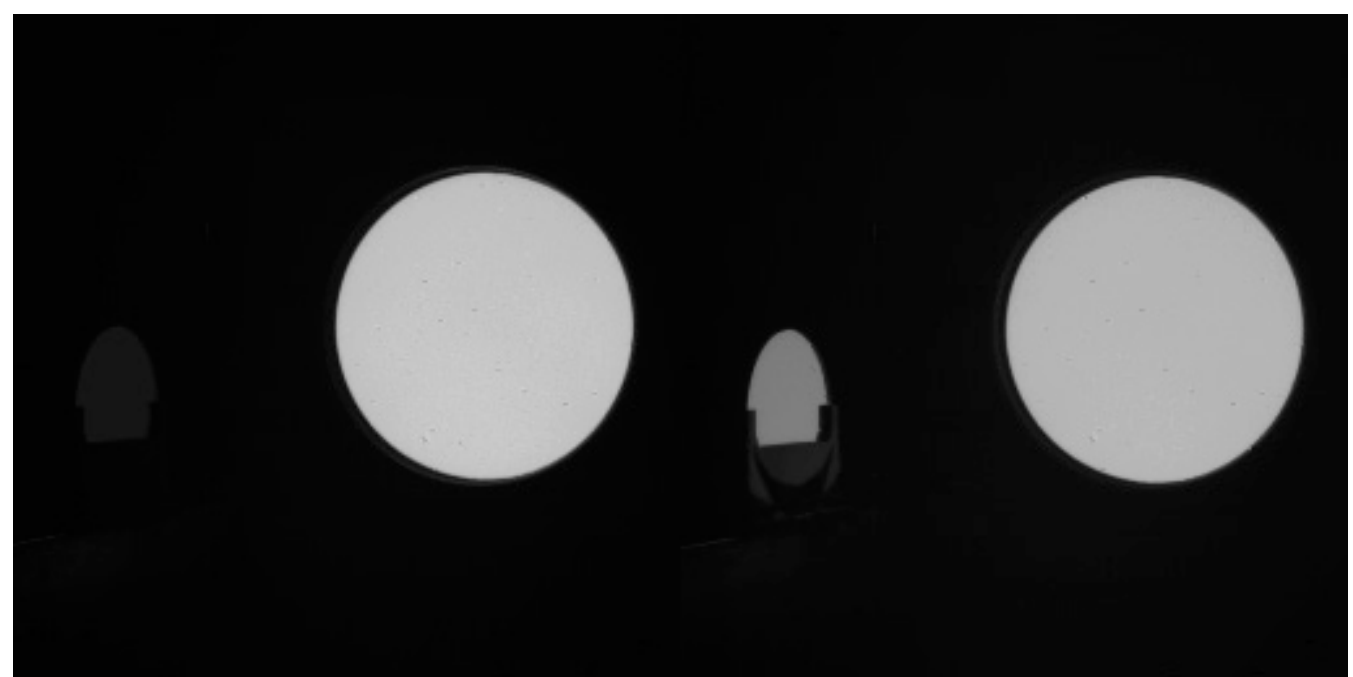

Fig. 6: Infrared Image of a Black Body @ $150^{\circ} \mathrm{C}$ (big sphere) and the reflected radiation from a polished silicon-wafer (small sphere) through a polarizer. On the left the polarizer is adjusted for a minimum intensity of reflection intensity and on the right for a maximum. 


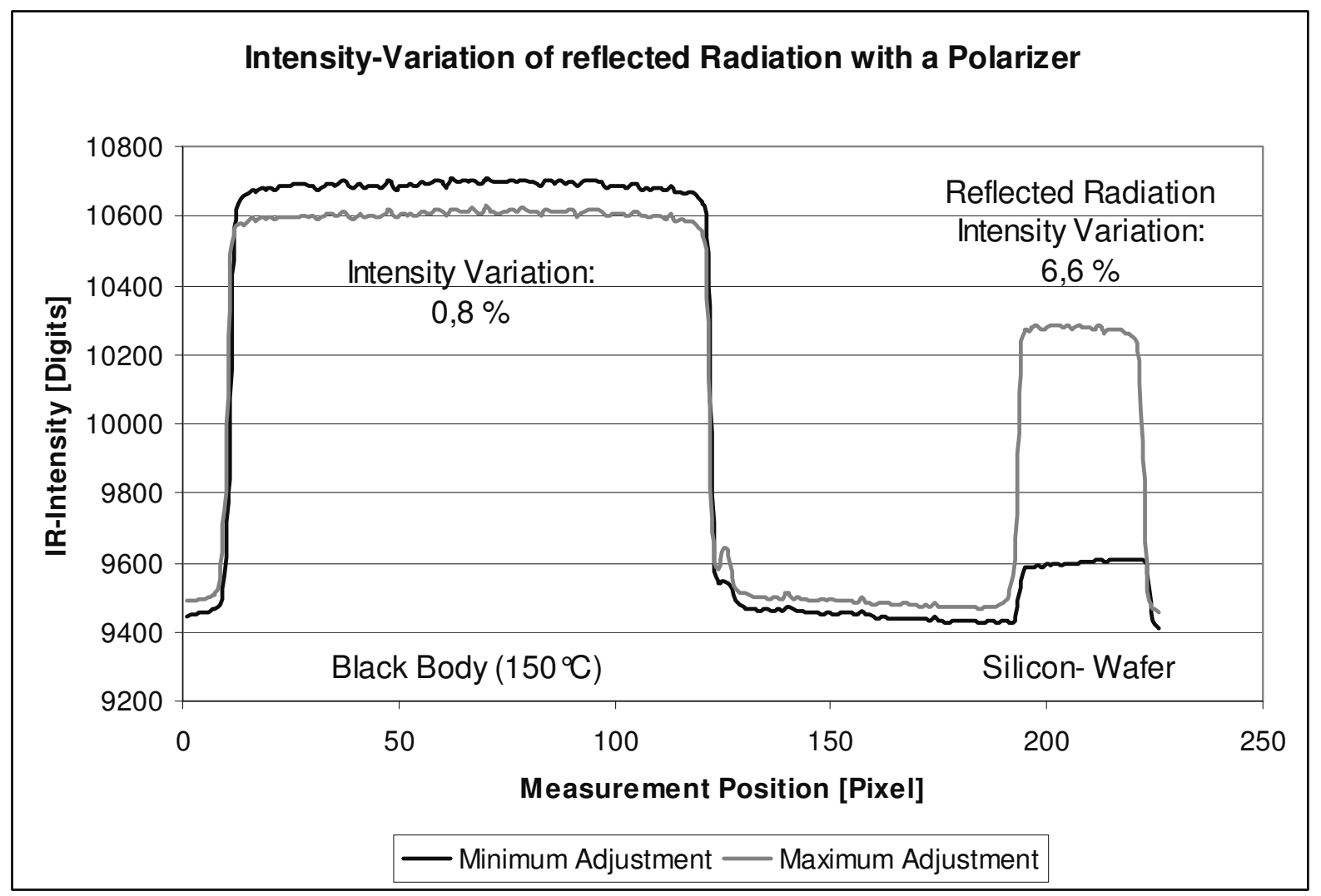

Fig. 7: Measured Intensity along a line of interest through the Black Body and the silicon-wafer.

\section{Summary and outlook}

In this paper, a method is shown which enables us to infinitely adjust the attenuation of the infrared radiation emitted by an object observed by an infrared camera. The used new kind of polarizers, which are based on silver nano-particles, show good results. The existing measurement range of the tested camera could thus be greatly increased, in that an overcharging of the sensor does not occur. Furthermore the method allows us to observe objects with high temperature differences, i.e. at cutting processes. Regions with a high radiation level, which can cause an overcharging of the detector and are interesting for measurement, can be shielded by a radiation stop. Hereby the blooming effect will be almost disabled. To avoid the change of the integration time, it should be matched to the lower temperature range. The high temperature range can then be attenuated so that it fits into the range, as selected by the integration time.

It was also shown that nuisance reflections could be reduced by this polarizer.

Further works shall examine the advantages and disadvantages of comparing different types of polarizers as the wire grid type. Also the effect of the transfer function from the polarizer to the temperature measurement behaviour will be of interest. Finally the attenuator cell should be tested in a cutting process. 